in Granada (ibs. GRANADA), Biohealth Research Institute in Granada (ibs. GRANADA), Granada, Spain: ${ }^{3}$ Unidad de Enfermedades Autoinmunes Sistémicas, Servicio de Medicina Interna, Hospital Universitario San Cecilio. Unidad de Enfermedades Autoinmunes Sistémicas, Servicio de Medicina Interna, Hospital Universitario San Cecilio., Granada, Spain; ${ }^{4}$ University of Granada, Department of Medicine. Faculty of Medicine. University of Granada, Granada, Spain

Background: Osteoporosis is a common comorbidity in patients with systemic lupus erythematosus (SLE). Available evidence showed that autoimmunity and associated inflammation play main effect in the pathogenesis of negative skeletal effects in SLE patients. However, the potential contribution of disease-associated factors to bone status in SLE is not well known since the reported risk factors from different studies differ greatly.

Objectives: The aim of this study was to examine frequency of reduced bone mass in SLE women, and determine their potential associations with disease activity, damage accrual and SLE-related clinical markers.

Methods: A cross-sectional study including a total 121 Caucasian pre-menopausal and postmenopausal women was conducted (mean age $49.29 \pm 12.43$ years). The SLE Disease Activity Index (SLEDAI-2K) and the SDI Damage Index were used to asses disease activity and disease-related damage, respectively. Bone mineral density (BMD) of the left femoral neck and lumbar spine (L2-L4) were measured by dual-energy X-ray absorptiometry (Hologic QDR 400).

Results: Ten patients (8.3\%) had osteoporosis, $63(52.1 \%)$ patients had osteopenia and $6.8 \%$ of women had history of previous fracture. Patients with low bone mass had a significantly higher mean SDI $(1.36 \pm 1.26$ versus $0.70 \pm 1.09 p=0.003)$. T-score at lumbar spine was inversely correlated with SDI score $(r=-0.222$, $p=0.014)$ and complement C3 level $(r=-0.206, p=0.024)$. Results of bivariate correlations showed that T-score at lumbar spine was inversely correlated with SDI score $(r=-0.222, p=0.014)$ and complement C3 level $(r=-0.206, p=0.024)$. SDI scores were significantly different between patients with osteoporosis, osteopenia and normal BMD after adjusting for age, menstrual status, BMI, time since diagnosis and corticoid use $(p=0.004)$

Conclusion: There is a high prevalence of low BMD in Caucasian women with SLE and this status of osteopenia/osteoporosis was associated with higher damage accrual scores, supporting that disease damage may itself be a major contributor to the low BMD. SLE women with organ damage require regular bone status monitoring to prevent further musculoskeletal damage. Since diminished BMD is a main comorbidity it is therefore essential to study, monitor and prevent osteoporosis in SLE women to avoid fractures leading to reduced quality of life. REFERENCES:

[1] Wang X, Yan S, Liu C, Xu Y, Wan L, Wang Y, et al. Fracture risk and bone mineral density levels in patients with systemic lupus erythematosus: a systematic review and meta-analysis. Osteoporos Int 2016;27:1413-23.

[2] Mendoza-Pinto C, Rojas-Villarraga A, Molano-Gonzalez N, Jimenez-Herrera EA, De La Luz Leon-Vazquez M, Montiel-Jarquõn A, et al. Bone mineral density and vertebral fractures in patients with systemic lupus erythematosus: $A$ systematic review and meta-regression. PLoS One 2018;13:1-15.

[3] Xia J, Luo R, Guo S, Yang Y, Ge S, Xu G, et al. Prevalence and Risk Factors of Reduced Bone Mineral Density in Systemic Lupus Erythematosus Patients: A Meta-Analysis. Biomed Res Int 2019;2019.

[4] Carli L, Tani C, Spera V, Vagelli R, Vagnani S, Mazzantini M, et al. Risk factors for osteoporosis and fragility fractures in patients with systemic lupus erythematosus. Lupus Sci Med 2016;3:1-5.

Acknowledgements: This research was supported by the grant PI0523-2016 from "Consejería de igualdad, salud y políticas sociales" (Junta de Andalucía) and is part of the research group LyDIMED "Lupus y Dieta Mediterránea" Disclosure of Interests: None declared

DOI: 10.1136/annrheumdis-2021-eular.2011

\section{AB0316 ADHERENCE TO THE MEDITERRANEAN DIET AND ITS ASSOCIATION WITH DISEASE ACTIVITY AND DAMAGE ACCRUAL IN WOMEN WITH SYSTEMIC LUPUS ERYTHEMATOSUS}

M. Correa Rodríguez ${ }^{1,2}$, G. Pocovi-Gerardino², J. L. Callejas-Rubio ${ }^{2,3}$, R. RíosFernández $z^{2,3}$, M. Martín Amada ${ }^{4}$, M. Cruz-Caparrós ${ }^{5}$, B. Rueda-Medina ${ }^{1,2}$, N. Ortego ${ }^{2,6} .{ }^{1}$ University of Granada, Nursing, Granada, Spain; ${ }^{2}$ Biohealth Research Institute in Granada (ibs.GRANADA), Biohealth Research Institute in Granada (ibs. GRANADA), Granada, Spain; ${ }^{3}$ Unidad de Enfermedades Autoinmunes Sistémicas, Servicio de Medicina Interna, Hospital Universitario San Cecilio., Unidad de Enfermedades Autoinmunes Sistémicas, Granada, Spain; ${ }^{4}$ Unidad de Enfermedades Autoinmunes Sistémicas. Servicio de Medicina Interna. Complejo Hospitalario de Jaén, Spain., Unidad de Enfermedades Autoinmunes Sistémicas., Jaén, Spain; ${ }^{5}$ Unidad de Enfermedades Autoinmunes Sistémicas. Servicio de Medicina Interna. Hospital de Poniente, El Ejido, Spain, Unidad de Enfermedades Autoinmunes Sistémicas, Almería, Spain; ${ }^{6}$ University of Granada, Department of Medicine.
Faculty of Medicine. University of Granada. Avenida de la Investigación, 11, 18016 Granada, Spain., Granada, Spain

Background: There is limited evidence regarding the impact of the Mediterranean Diet (Med Diet) on autoimmune diseases although it has been proposed that adherence to the Med Diet may decrease the risk of certain autoimmune diseases. However, the potential usefulness of the Med Diet as a high-quality dietary pattern for other autoimmune diseases such as SLE has not yet been investigated.

Objectives: The aim of this study was to evaluate the potential association between the adherence to the Med Diet and disease activity, damage accrual and SLE-related clinical markers in a large cohort of women with SLE.

Methods: In this cross-sectional study, we assessed Med Diet adherence through a 14-item questionnaire in 253 women with SLE (mean age $46.74 \pm$ 12.70 years). The SLE Disease Activity Index (SLEDAI-2K) and the SDI Damage Index were used to asses disease activity and disease-related damage, respectively. Levels of C-reactive protein (CRP; mg/dL), homocysteine (Hcy; mol/L), anti-double stranded DNA antibodies (anti-dsDNA) (IU/mL), complement $\mathrm{C} 3$ $(\mathrm{mg} / \mathrm{dL})$, and complement $\mathrm{C} 4(\mathrm{mg} / \mathrm{dL})$ were determined.

Results: The Med Diet adherence score was classified as follows: low adherence ( $\leq 5$ points); medium adherence (6-9 points) and good adherence ( $\geq 10$ points). Only $50.2 \%$ of the SLE women had good adherence to the Med Diet. The ANCOVA models showed significant differences between patients with good adherence to the Med Diet and those with medium and low adherence in SLEDA $(p \leq 0.001)$ and SDI $(p \leq 0.001)$. Age, medical treatment (immunosuppressors, corticoids, or antimalarials), smoking status, and body mass index (BMI) were included as confounding factors. The odds ratio (OR) for having active SLE (SLE$D A I \geq 5)$ or the presence of damage (SDI $\geq 1$ ) was lower among patients whose Med Diet score was higher $(p \leq 0.001)$.

Conclusion: Adherence to the Med Diet influences disease activity and damage accrual in SLE women. Thus, these patients would benefit from nutritional counselling and education on modification, to help adapt their lifestyles towards the Med Diet pattern. This would help slow the progression of SLE and the damage it causes.

\section{REFERENCES:}

[1] Sedaghat F, Jessri M, Behrooz M, Mirghotbi M, Rashidkhani B. Mediterranean diet adherence and risk of multiple sclerosis: a case-control study. Asia Pac J Clin Nutr. 2016;25(2):377-84.

[2] orsyth C, Kouvari M, D'Cunha NM, Georgousopoulou EN, Panagiotakos DB Mellor DD, et al. The effects of the Mediterranean diet on rheumatoid arthritis prevention and treatment: a systematic review of human prospective studies. Rheumatol Int. 2018 May 18;38(5):737-47.

[3] Minihane AM, Vinoy S, Russell WR, Baka A, Roche HM, Tuohy KM, et al. Low-grade inflammation, diet composition and health: current research evidence and its translation. Br J Nutr. 2015 Oct 14;114(7):999-1012.

Acknowledgements: This research was supported by the grant PI0523-2016 from "Consejería de igualdad, salud y políticas sociales" (Junta de Andalucía) and is part of the research group LyDIMED "Lupus y Dieta Mediterránea". Disclosure of Interests: None declared

DOI: 10.1136/annrheumdis-2021-eular.2054

\section{AB0317 $\quad$ ADHERENCE TO MEDITERRANEAN DIET AND NUTRITIONAL STATE IN ITALIAN WOMEN WITH ISOLATED SJÖGREN'S SYNDROME}

R. Izzo ${ }^{1}$, S. Colafrancesco', A. Pinto ${ }^{2}$, A. Gattamelata ${ }^{1}$, F. Giardina ${ }^{1}$, F. Claudia ${ }^{2}$, L. M. Donini ${ }^{2}$, R. Priori ${ }^{1}{ }^{1}$ Sapienza Università di Roma, Dipartimento di Medicina interna e Specialità Mediche, Roma, Italy; ${ }^{2}$ Sapienza Università di Roma, Dipartimento di Medicina Sperimentale, Roma, Italy

Background: The Mediterranean Diet (MD) has anti-inflammatory and immunomodulatory effects ${ }^{1,2}$ suggesting a protective role in rheumatic diseases. There is limited knowledge about the eating habits and the nutritional state in patients with isolated Sjögren Syndrome (SS) living within the Mediterranean area.

Objectives: assessment of adherence to the MD and analysis of the nutritional state in women with SS and their correlations with the clinical, laboratory and histological data of the disease.

Methods: patients classified as isolated SS according to AECG criteria $2002^{3}$ who had undergone to minor salivary gland biopsy during the previous twelve months were consecutively enrolled during follow-up visits. The adherence to the MD was assessed by the Med Diet (MDiet) ${ }^{4}$ which includes eleven groups of foods; to each group is assigned a value ranging between 0 and 5 based on the frequency of monthly intake. The total score spans from 0 (poor adherence) to 55 (maximum adherence). The level of physical activity was measured by the 6-minutes walking test (6MWT) and by the International Physical Activity Questionnaire (IPAQ). Systemic disease activity was evaluated with the EULAR SS disease activity index (ESSDAI); EULAR SS patient-reported index (ESSPRI) was calculated as well. A subgroup of patients was asked to fill a daily food 
diary processed with FOOD CONS software which allows to study in detail their eating habits. Nutritional state, muscle strength and basal metabolic rate were assessed. Alcoholism or drug abuse, diabetes mellitus, specific dietary models, treatment with drugs and/or food supplements with anti-inflammatory and/or antioxidant activity were considered exclusion criteria. Multivariate linear regression was performed with $\mathrm{R}$ project for Statistical Computing. RESULTS:

\section{$\mathrm{N}=40 \mathrm{~N}=26$}

Age, median (range) $53(25-80) 33$ (25-71)

BMI, median (range) 21 (19-29.3) 25.1 (19-33.7)

MedDiet score, median (range) 33 (26-43) $33(23-40)$

ESSDAI, median (range) $2(0-16) 1(0-16)$

ESSPRI, median (range) 6 (0-8.6) $5.3(1.6-9)$

ESSPRI dryness, median (range) $6(0-10) 6(2-10)$

Focus score, median (range) 2.5 (0-9.6) $1.7(0.8-6.24)$

ASM kg, median (range) - 16.8 (13.3-21.7)

IPAQ meters, median (range) - 1386 (99-11865)

6MWT meters, median (range) - 595 (536-680)

BMI, body mass index; ASM appendicular skeletal mass; IPAQ International Physical Activity Questionnaire; 6MWT, six minute walking test.

MDiet was administered to 40 female SS outpatients. Even if not reaching significativity, patients with a higher focus score in their MSG have a lower value of MDiet score $(p=0.058, r=-1.00)$. The MDiet score is not associated with ESSDAI ( $p=0.85, r 0.02)$, but only with lower serum levels of C3 $(p=0.004, r=-0.08)$. In 26 patients, daily food questionnaire shows that their diet consists of $43 \%$ of carbohydrates while fats represent $40 \%$ of total energy intake, the remaining $17 \%$ daily energy comes from proteins. Fat consumption is higher compared to the levels of energy and nutrient intake for the Italian population ${ }^{5}$. Six patients had a reduction in muscle mass; sarcopenia is not associated to ESSDAI ( $p=0.610)$. The MDiet score and the amount eaten of polyunsaturated fatty acids (PUFA) were reduced in patients with high value of subscale dryness of ESSPRI ( $p=$ $0.057, r-1.21 ; p=0.610, r-1.00$ )

Conclusion: This study highlights a lower degree of glandular lymphocytic infiltration (expressed as focus score) in minor salivary glands in patients following MD, so its anti-inflammatory role of seems to be confirmed. SS patients have an unbalanced diet because of a higher intake of fat foods, likely for their lubricating effect. Despite the absence of correlation with objective parameters, the increased dryness in patients with a reduced intake of PUFA arouses our interest in a future study including omega-3 supplementation.

REFERENCES:

[1] Schwingshackl L et al., Nutr Metab Cardiovasc Dis 2014

[2] Mena MP et al., Am J Clin Nutr. 2009

[3] Vitali C et al, ARD 2002

[4] Panagiotakos D et al., J Med Food 2007

[5] LARN 2014

Disclosure of Interests: None declared

DOI: 10.1136/annrheumdis-2021-eular.2154

\section{AB0318 2 RISK FOR CONCOMITANT AUTOIMMUNITY IN PATIENTS WITH ANTIPHOSPHOLIPID SYNDROME; A SWEDISH COHORT STUDY}

N. Karandyszowska ${ }^{1}$, J. Oesman ${ }^{1}$, H. Alagündüz ${ }^{1}$, M. Magnusson ${ }^{2}$, E. Svenungsson ${ }^{1}$, M. Bruzelius ${ }^{1}$, A. Antovic ${ }^{3} .{ }^{1}$ Karolinska Institutet, Department of Medicine Solna, Stockholm, Sweden; ${ }^{2}$ Karolinska Institutet, Clinical Chemistry and Blood Coagulation, MMK, Stockholm, Sweden; ${ }^{3}$ Karolinska Institutet, Department of Medicine Solna, Division of Rheumatology, Stockholm, Sweden

Background: In patients with the antiphospholipid syndrome (APS), concomitant systemic autoimmune rheumatic diseases (SARD) are common and often associated with more disease associated damage.

Less is known about the prevalence of non-rheumatic autoimmune diseases (NRAID) in patients with APS.

Objectives: To evaluate the incidence and prevalence of concomitant autoimmune diseases (AID) in a cohort of APS patients. The risk of AID was also evaluated with respect to the antiphospholipid antibodies (aPL) profiles.

Methods: This retrospective cohort study comprises consecutive patients identified with APS through review of electronic medical records at Karolinska University Hospital, Sweden between 2014 and 2020. Exclusion criteria were misdiagnosis and age $<18$. Descriptive statistics was used for baseline data and multivariable Cox proportional hazard regression analysis to investigate the risk factors to develop new onset AID. Ethical approval was obtained from the Swedish Ethical Review Authority (2020-02333).

Results: Of 271 included patients, $66 \%$ were women and the median age at diagnosis of APS was 43 years (IQR 31-55). At inclusion, $130(48 \%)$ patients presented with other AID; 101 (37\%) of them had a concomitant SARD while 54 $(19 \%)$ had a NRAID. Systemic lupus erythematosus (SLE) was the most frequent in $30 \%$ of patients, followed by autoimmune thyroid disease (ATD) in $10 \%$ of patients.

In addition, 35 (13\%) APS-patients developed AID during the study period, corresponding to an incidence rate of $28.4(95 \% \mathrm{Cl} ; 19.3-40.3)$ per 1.000 person-years with mean time at risk of $4( \pm 2)$ years. Twenty-one $(8 \%)$ patients developed a SARD and further $14(5 \%)$ were diagnosed with a NRAID.

The cumulative incidence for AID was significantly higher in patients with high titers of IgG aPL. Patients that developed SARD had significantly higher median titers of a- $\beta 2$ GPI IgG isotype, $p=0.05$. In the NRAID group, median $a-\beta 2 G P I$ and $\mathrm{aCL} \lg \mathrm{G}$ isotypes were significantly increased, $\mathrm{p}=0.02$ and $\mathrm{p}=0.04$, respectively. The hazard ratio to develop diagnosis of AID was significantly increased in patients with high titers of the IgG isotype aPL (HR $2.495 \% \mathrm{Cl}$; 1.1-5.3). Obstetric APS manifestations were associated with a significantly increased hazard ratio of 2.8 (95\% Cl; 1.1-7.7) to develop $S A R D$, and also trendwise for $A I D$, as a compound variable.

During the study period, 52 patients had at least one new APS manifestation, as defined by the Sydney criteria (1). In comparison to patients without new manifestations, these patients had significantly higher median titers of aPL of the IgG isotype, and concomitant AID at first visit $(p=0.01, p=0.02$, respectively).

Conclusion: APS patients are at high risk to develop other AID, and APS patients with concomitant AID had an increased risk to develop new clinical APS manifestations. These findings might be helpful when considering risk stratification and alternate treatment options in this patient group.

\section{REFERENCES:}

[1] Miyakis S, Lockshin MD, Atsumi T et al. International consensus statement on an update of the classification criteria for definite antiphospholipid syndrome (APS). J Thromb Haemost 2006; 4: 295-306.

Acknowledgements: I have no acknowledgements to declare.

Disclosure of Interests: None declared

DOI: 10.1136/annrheumdis-2021-eular.2194

\section{$\mathrm{AB} 0319$ \\ HEALTH-RELATED QUALITY OF LIFE ASSESSED BY LUPUSQOL IN 1102 LUPUS PATIENTS FROM RUSSIAN FEDERATION (RF), KIRGHIZSTAN AND KAZAKHSTAN (RENAISSANCE COHORT)}

E. Aseeva ${ }^{1}$, S. Solovyev ${ }^{1}$, G. Koilubaeva ${ }^{2}$, B. Issayeva ${ }^{3}$, M. Saparbayeva ${ }^{3}$, N. Nikishina ${ }^{4}$, A. Mesnyankina ${ }^{4}$, A. Lila ${ }^{1} .{ }^{1}$ Rheumatology Federal State Research Institution (FSRI) named after V. A . Nasonova, Intensive Care Department, Moscow, Russian Federation; ${ }^{2}$ Rheumatology, National Center of Cardiology and Internal Medicine named after Academician M. Mirrahimov (NCCIM), Rheumatology Department, Bishkek, Kyrgyzstan; ${ }^{3}$ Asfendiyarov National Medical University, City Rheumatology Center, Almaty, Kazakhstan ${ }^{4}$ Rheumatology Federal State Research Institution (FSRI) named after V. A. Nasonova, Intensive Care Department, Moscow, Russian Federation

Background: Health related quality of life (HRQoL) is an aspect that is generally underexplored in the routine clinical practice but it is attracting more and more attention. Lack of concordance between physician derived outcomes of disease activity and patient HRQoL outcomes is commonly found in SLE. This is due to the fact that the former is influenced by the immunologic pathogenic inflammatory mechanisms while the latter may be affected by a variety of psychological, social and co-morbid conditions.

Objectives: The aim of this study was to compare health-related quality of life assessed by LupusQoL in 1102 Lupus patients (pts) from the Russian Federation, Kirghizstan and Kazakhstan (RENAISSANCE cohort).

Methods: 1102 SLE pts who fulfilled SLICC 2012 criteria were enrolled into the study. The SLEDAI 2K index activity, SLICC damage index, and HRQoL using the LupusQoL questionnaire (validated for the Russian-speaking population in 2018) were evaluated. Descriptive statistics, Spearman's correlation coefficients were performed to analyze the data.

Results: 400/600/102 Lupus pts from RF, Kirghizstan and Kazakhstan respectively were studied. The pts were predominantly female $(91 \% / 93 \% / 98 \%)$ and of indigenous nationality (Russian $83 \% /$ Kirghiz $88 \% /$ Kazakh $76 \%$ ) with a mean $\pm S D$ age of $34,2 \pm 11,5 / 35,07 \pm 12,2 / 33,8 \pm 10,5$ years $(p=0,36 ; p=0,96)$ respectively. The mean disease duration $(\mathrm{Me})$ in $\mathrm{RF} /$ Kazakhstan was 6 [3;12] $5[2 ; 9]$ years $(p=0,15)$, in Kirgizstan $-2[0,6 ; 7]$ years $(p \leq 0,05)$. Kazakh pts $(p=0,00)$ have higher disease activity (SLEDAI $2 \mathrm{~K}$ ) $17,6 \pm 8,8$ than Kirghiz pts $15,1 \pm 8,3$ and Russian pts $9,4 \pm 8,1$. SLICC damage index $2,39 \pm 1,61$ in Kazakh pts $(p=0,00)$ was higher than in Russian 1,57 $\pm 1,54$ and Kirghiz $0,79 \pm 0,32$ pts. $54 \%$ pts from RF and $58 \%$ from Kazakhstan had high education. Only 33\% pts from Kirgizstan also had high education. $45 \% / 24 \% / 33 \%$ pts from RF, Kirgizstan and Kazakhstan respectively had steady employment, $53 \% / 30 \% / 32 \%$ pts received disability benefits. HRQoL assessed by LupusQoL was low in all patients with SLE. The mean scores for each of the domains of LupusQoL are shown in Table 1. The statistically significant lowest HRQoL in all 8 domains is obtained among 